\title{
Évaluation de la résistance au bayoud (Fusarium oxysporum f sp albedinis) chez le palmier dattier. Comparaison de méthodes d'inoculation expérimentale en palmeraie et en pépinière
}

\author{
My H Sedra
}

INRA, centre régional du Haouz-Présahara, laboratoire de pathologie végétale-mycologie, BP 533, Marrakech, Maroc

(Reçu le 12 novembre 1992; accepté le 12 août 1994)

\begin{abstract}
Résumé - Pour combattre le bayoud causé par Fusarium oxysporum $\mathfrak{f}$ sp albedinis (Malençon) $\mathrm{Sn}$ et $\mathrm{H}$, la sélection de palmiers productifs, de bonne qualité dattière et résistants à la maladie est jusqu'ici la voie privilégiée, qui nécessite une méthodologie rigoureuse. Les techniques nouvelles directement liées à l'appréciation du potentiel infectieux des sols et à l'étude du comportement des cultivars et clones du matériel jeune ou adulte, voire des vitroplants, ouvrent de nouvelles perspectives de sélection beaucoup plus rapide et en milieu contrôlé. Dans cette étude, la comparaison des niveaux d'infectivité et de réceptivité de 10 échantillons de sol du domaine expérimental de Zagora a mis en évidence des différences significatives de comportement de ces sols vis-à-vis du bayoud. En outre, il ressort la nécessité d'une inoculation artificielle en sol de même niveau de réceptivité au bayoud. Elle favorise le processus d'infection et permet d'accélérer la sélection des arbres à tester pour leur résistance. La méthode d'inoculation la plus facile à appliquer et à maîtriser consiste à apporter l'inoculum liquide par injection au pied de l'arbre au moyen de tuyaux. Cette méthode testée en palmeraies et sur jeunes palmiers en sachets (pépinière) semble la plus efficace pour provoquer la fusariose bayoud sur les palmiers sensibles.
\end{abstract}

palmier dattier / fusariose (bayoud) / sélection / résistance

\begin{abstract}
Summary - Evaluation of the resistance of date palm cultivars to bayoud disease (Fusarium oxysporum $f \mathrm{sp}$ albedinis). Comparison of experimental inoculation methods in the field and nursery. In order to control bayoud disease due to Fusarium oxysporum $f s p$ albedinis (Malençon) Sn et $H$, the selection of date palm trees on the basis of resistance and fruit quality criteria has up to now been the best use of a method that requires a rigorous methodology. New methods to evaluate the inoculum potential and the resistance or susceptibility cultivars and clones at early or late stages or on vitroplants allow new methods of rapid selection. In this study, the comparison of the infectivity and receptivity levels of 10 sampled soils from the Experimental Station of Zagora showed significant differences in the soil behaviour towards bayoud disease. Moreover, the artificial inoculation of the date palm trees in soil with homogeneous receptivity levels (in the field) is essential. It favours the infection process and allows an acceleration of the selection of the trees tested for their resistance. In the field, the inoculation method presents an easy and controlled application, and consist of bringing the liquid inoculum, through pipes, to the roots via an injection beside the palm tree. This method has been tested in the field and nursery on the young palm tree, and is the most efficient method to cause bayoud disease on susceptible palm trees.
\end{abstract}

date palm / Fusarium wilt / selection / resistance 


\section{INTRODUCTION}

La fusariose vasculaire du palmier dattier, dénommée communément bayoud, est causée par Fusarium oxysporum $f \mathrm{sp}$ albedinis (Malençon ) Sn et H. Jusqu'ici, plus de 13 millions de palmiers ont été détruits par cette maladie au Maroc et en Algérie (Pereau-Leroy, 1958 ; Djerbi, 1988). Le bayoud fait partie des maladies d'origine tellurique contre lesquelles il est difficile de lutter. La sélection des cultivars résistants est la voie qui a été privilégiée au Maroc pour lutter contre cette maladie. Les 6 cultivars marocains actuellement sélectionnés pour leur résistance (Pereau-Leroy, 1958 ; Louvet et Toutain, 1973 ; Saaidi et al, 1981) ne produisent malheureusement que des fruits de qualité inférieure à celle des cultivars commerciaux sensibles à la maladie. De ce fait, la diffusion de ces cultivars résistants se trouve freinée.

En attendant les résultats de la sélection entreprise sur les descendances de croisements contrôlés, les populations naturelles des "Khalts" issus de semis spontanés de graines, représentent une source potentielle de cultivars pouvant réunir résistance à la maladie et bonne qualité dattière des fruits.

La palmeraie marocaine possède aujourd'hui, un effectif de «Khalts» estimé à environ 2,7 millions d'individus, représentant près de $55 \%$ de l'effectif total de la population phoénicicole nationale. La sélection massale des palmiers issus de semis naturel, sur le critère de la qualité des dattes, a permis entre les années 1979 et 1983 de repérer en palmeraie 2337 «khalts» de bonne à excellente qualité dattière (Djerbi et al, 1986). La mise au point de méthodes fiables portant sur une évaluation de la résistance au bayoud de ce patrimoine génétique naturel devrait permettre, à court terme, de sélectionner des clones de palmier associant les 2 critères recherchés (Sedra, $1990 ; 1992 ; 1993 b)$.

Si les qualités agronomiques du matériel marocain et étranger sont relativement bien connues, le programme de sélection pour la résistance au bayoud a, en revanche, nécessité un travail méthodologique préalable.

Plusieurs auteurs s'accordent à constater que la population naturelle de $F$ of $\mathrm{sp}$ albedinis est généralement faible dans les sols de palmeraie et que sa répartition spatiale est très hétérogène (Bulit et al, 1967 ; Louvet et al, 1970 ; Djerbi et al, 1985). Dans 15 sols de palmeraies, nous avons trouvé 0 à 106 propagules par g de sol (Sedra, 1985). Les analyses préliminaires relatives aux sondages effectués dans les sols du domaine expérimental de Zagora (INRA) ont montré qu'il était difficile de détecter le parasite dans certains échantillons de terre. Le niveau et la dynamique du potentiel infectieux des sols des sites expérimentaux de Zagora (INRA) et d'Errachidia (ORMVAT) où s'effectue la sélection sont donc insuffisamment connus.

De plus, Sedra et Rouxel (1989) et Sedra et al (1994) ont montré que certains sols de palmeraies présentent des niveaux de réceptivité différents vis-à-vis du bayoud. De ce fait, il est difficile de tester de manière fiable le comportement d'un matériel génétique à l'égard du bayoud dans les conditions d'infestation naturelle au champ. Les risques sont encore accrus dans le cas des «khalts" dont chaque génotype n'est représenté que par un nombre très limité d'individus, mais aussi dans les palmeraies aux sols hétérogènes sur le plan de leur réceptivité à la maladie.

D'autre part, la diversité des souches du parasite présentes dans un sol infesté peut influencer le comportement à l'égard des cultivars testés. En effet, de grandes différences ont été observées dans l'agressivité des isolats prélevés à partir des palmiers atteints plantés en différents endroits du domaine expérimental de Zagora (Sedra, 1993a). En outre, les rares attaques du bayoud que nous avons décelées en 1989 en palmeraie et au domaine de Zagora sur 3 cultivars parmi les 6 considérés comme totalement résistants (Saaidi et al, 1981) laissent penser à l'adaptation de nouvelles souches du parasite sur ces cultivars.

Par ailleurs, les résultats préliminaires obtenus sur l'évaluation du comportement de quelques cultivars marocains et irakiens au stade rejets plantés en hors-sol (Djerbi et Sedra, 1982) montrent la nécessité d'améliorer cette méthode d'appréciation de la résistance. De ces observations et constatations sur le terrain ressort la nécessité de mettre au point une méthode fiable d'inoculation artificielle permettant de tester le comportement du palmier dattier vis-àvis de bayoud, tant en palmeraie qu'en pépinière. Pour ce faire, nous avons procédé en 3 étapes:

- nous avons d'abord vérifié l'influence du potentiel infectieux et de la réceptivité du sol sur l'appréciation de la sensibilité de palmier dattier à la maladie en palmeraie ;

- nous avons ensuite testé l'intérêt de l'inoculation artificielle pour la sélection de clones résistants à la maladie ; 
- nous avons enfin étudié la fiabilité de quelques méthodes d'inoculation artificielle de palmiers adultes au champ et de jeunes palmiers élevés en sachet plastique sous abri ombragé.

\section{MATÉRIEL ET MÉTHODES}

\section{Évaluation du potentiel infectieux et de la réceptivité des sols à la fusariose vasculaire}

Cette étude a porté sur 10 échantillons de sol prélevés dans 10 blocs différents (environ 0,5 ha chacun) d'un ancien foyer de bayoud du domaine expérimental de Zagora. Chaque échantillon est constitué d'un regroupement de 5 sous-échantillons de $4 \mathrm{~kg}$ de sol chacun, distants de $10 \mathrm{~m}$, prélevés sur les diagonales de chaque bloc et à une profondeur comprise entre 20 et $60 \mathrm{~cm}$, couche de sol au niveau de laquelle le palmier développe un système racinaire important (Toutain, 1977). Chaque échantillon de sol est infesté avec des doses croissantes $\left(0,10^{2}, 10^{3}, 5 \times 10^{3}, 10^{4}\right.$ propagules par $\mathrm{g}$ de sol) d'un inoculum de $F$ of $\mathrm{sp}$ albedinis (isolat Foa 133) produit selon la technique décrite par Tello-Marquina et Alabouvette (1984). Cet isolat est reconnu très agressif sur les plantules issues de graines (Sedra, 1993b). La terre est répartie en pots de $1000 \mathrm{ml}$ et chaque traitement comporte 6 pots ensemencés avec 5 graines prégermées issues d'un croisement réalisé entre parents sensibles ( $\mathrm{cV}$ Boufeggous $x$ mâle ZS3). La proportion d'individus sensibles dans cette descendance a été estimée à $93 \%$ après inoculation artificielle avec le parasite. La germination des graines est obtenue préalablement sur du sable stérile maintenu humide à une température de $35^{\circ} \mathrm{C}$ pendant $10 \mathrm{j}$. Les plantules sont ensuite élevées en serre durant 12 mois. La gravité de la maladie est évaluée par dénombrement mensuel des plantes malades par échantillon de sol et par dose d'inoculum. Le potentiel infectieux naturel évalué à la dose 0 propagules par $\mathrm{g}$ de sol et la réceptivité appréciée aux autres doses sont exprimés en nombres de plantes mortes.
Les résultats expérimentaux ont été comparés par analyse de la variance suivie du test de Duncan après transformation angulaire pour les nombres de plantes atteintes. Les régressions des résultats des sols sur les doses d'inoculum ont aussi été comparées.

\section{Étude de l'influence de l'inoculation artificielle de jeunes palmiers au champ}

Les effets de l'inoculation artificielle sur l'expression de la maladie sont évalués à partir de 3 méthodes de contamination in situ. Ces expérimentations se sont déroulées dans le foyer bayoudé du domaine expérimental de Zagora sur le cultivar Jihel sensible à la maladie.

\section{Méthode dite du "fossé / broyat" (Fb)}

Elle consiste à creuser autour de chaque palmier, âgé de 3 ans, un fossé circulaire, profond de 40 à $50 \mathrm{~cm}$, à un rayon de $50 \mathrm{~cm}$ du stipe (fig 1).

Deux lots de palmiers sont répartis au hasard:

- l'un, de 73 arbres destinés à recevoir l'inoculation artificielle ;

- l'autre, de 55 arbres recevant une inoculation factice, sert de témoin.

Sur le premier lot, on procède suivant la méthode indiquée par Djerbi et Sedra (1982) :

- ouverture de fossés individuels au pied de chaque palmier :

- inoculation à l'aide d'un mélange d'isolats de Fof $\mathrm{sp}$ albedinis obtenus à partir d'un broyat de palmes contaminées par le bayoud. La concentration en parasite de ce broyat est estimée à 100 à 150 propagules par g de broyat. On mélange 1 à $1,2 \mathrm{~kg}$ du broyat avec une cinquantaine de $\mathrm{kg}$ de sable que l'on incorpore soigneusement à la terre destinée à reboucher le fossé.

Sur la terre du fossé refermé, on sème 300 à 400 graines de palmier du cultivar sensible Boufeggous afin de maintenir le parasite en activité par des infections ultérieures sur les jeunes plantules issues de ce semis.

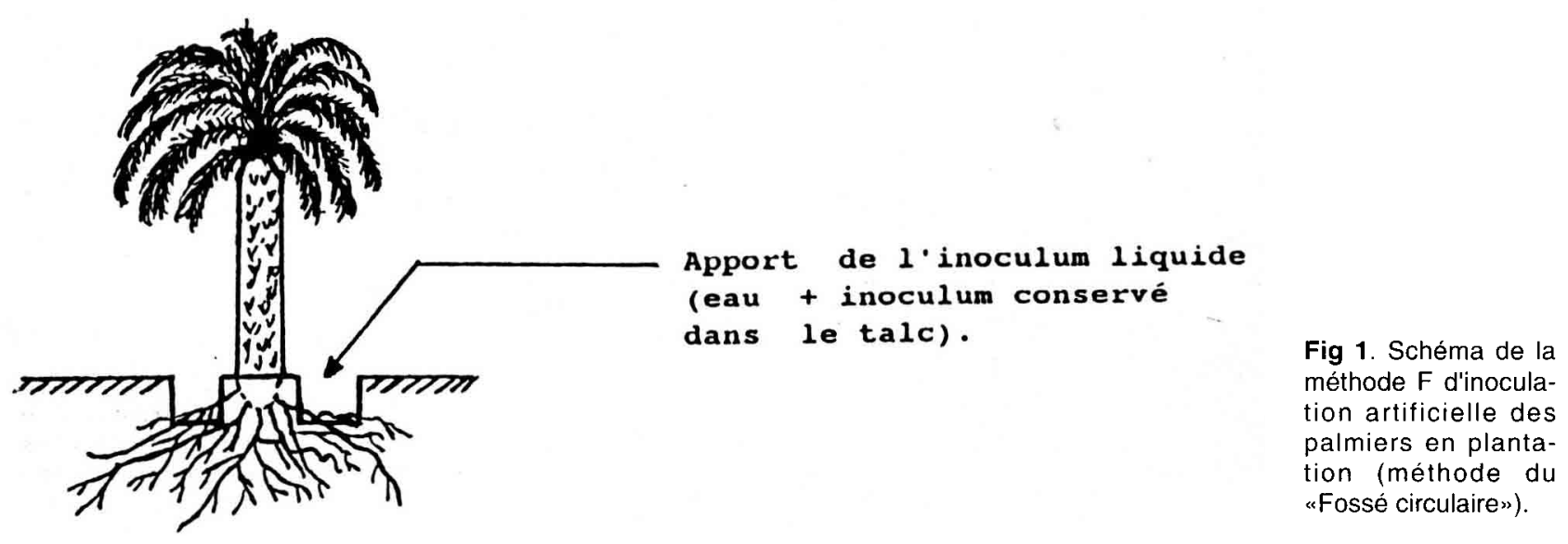


Sur le deuxième lot de palmiers, on creuse le même type de fossé individuel, que l'on rebouche immédiatement de la même façon mais sans procéder à l'inoculation.

Après 4 années d'observation, on dénombre, pour chacun des lots, le nombre de palmiers atteints par la maladie. La notation in situ est confirmée au laboratoire par le diagnostic des échantillons de palmes atteintes par le bayoud (tableau II).

\section{Méthode dite du «fossé/talc» (Ft)}

Cette expérimentation a débuté en 1986 et s'est déroulée sur la parcelle où l'essai fossé/broyat avait été mis en place 4 années auparavant. Les palmiers du cv Jihel sont maintenant âgés de 7 ans.

L'expérimentation est conduite sur les arbres restants des 2 lots précédemment définis lors de l'essai (Fb). Ces palmiers restants ne présentent aucun signe de contamination par la maladie. Sur le premier lot de 29 palmiers est pratiquée l'inoculation artificielle ; le deuxième lot de 37 palmiers sert de témoin.

Comme pour l'essai $(\mathrm{Fb})$ on pratique autour de chaque palmier un fossé aux dimensions définies en (Fb). La contamination artificielle du premier lot est réalisée à partir d'un inoculum du parasite (isolat Foa 133) sous la forme d'une suspension de chlamydospores conservées sur du talc à raison de $20 \mathrm{~g}$ de talc pour 20 I d'eau apportés par arbre traité. La concentration de l'inoculum est estimée à 5000 propagules par $\mathrm{ml}$ de suspension. Les 20 । de solution sont répartis dans le fossé entourant chaque arbre. Après infiltration le fossé est comblé.

L'incidence de la maladie sur les palmiers inoculés (Ft) ou non est notée tous les 3 mois pendant 4 années. Parallèlement, une contamination suivant la méthode Ft est appliquée sur 6 palmiers du cultivar résistant Bousthami noire (tableau III).

\section{Méthode dite du «tuyau» (T)}

Elle consiste à creuser, à environ $30 \mathrm{~cm}$ de part et d'autre de la base du stipe du palmier, 2 trous de $50 \mathrm{~cm}$ de diamètre et de 30 à $40 \mathrm{~cm}$ de profondeur selon la vigueur des plants, c'est-à-dire jusqu'à la zone supérieure des racines (fig 2). Chaque trou est d'abord partiellement comblé avec quelques pierres localisées au fond et recouvertes par environ $10 \mathrm{~kg}$ de sable. On introduit verticalement, à l'intérieur de ce substrat, la base d'un tuyau en polyéthylène rigide de $4 \mathrm{~cm}$ du diamètre et d'une longueur totale de $50 \mathrm{~cm}$ puis on procède au comblement avec la terre, en prenant soin de confectionner une cuvette du même rayon que les trous tout autour de chaque arbre.

Deux lots de 10 palmiers (cv Jihel), âgés de 5 ans, sont retenus pour cet essai.

Le premier lot est inoculé artificiellement. La veille de chaque irrigation gravitaire, chaque palmier de ce lot reçoit :

- à l'aide des 2 tuyaux laissés en place, 5 I d'inoculum liquide à la concentration de 5000 propagules par $\mathrm{ml}$ (préparation identique à celle décrite en $\mathrm{Ft}$ ) ;

- dans la cuvette d'irrigation 5 autres I d'inoculum. Ces apports sont hebdomadaires durant le mois de juin 1987.

Le deuxième lot de 10 palmiers ne reçoit pas d'inoculum mais la même quantité d'eau d'irrigation.

Le nombre de palmiers atteint par la maladie du bayoud est noté tous les 3 mois durant 4 années (tableau IV).

Les résultats obtenus pour les méthodes d'inoculation $\mathrm{Fb}, \mathrm{Ft}$, et $\mathrm{T}$ ont été comparés par le test $\chi^{2}$.

\section{Étude de l'efficacité de l'inoculation artificielle sur des rejets élevés en sachets sous abri}

Cette technique consiste à transplanter en mottes des rejets racinés de palmier d'un an et demi dans des sachets opaques en polyéthylène. Après 3 mois d'élevage sous abri ombragé, l'apport de l'inoculum sur les racines des jeunes plants est réalisé à l'aide d'un tuyau en polyéthylène rigide, placé à l'intérieur du substrat du sachet selon la technique précédemment décrite. L'inoculation artificielle est réalisée sur 2 lots de 10 rejets, appartenant au cv Jihel sensible et au cv Iklane très tolérant (Saaidi, 1992). Les jeunes palmiers servant de témoins ne reçoivent pas d'inoculum mais un apport identique en eau.

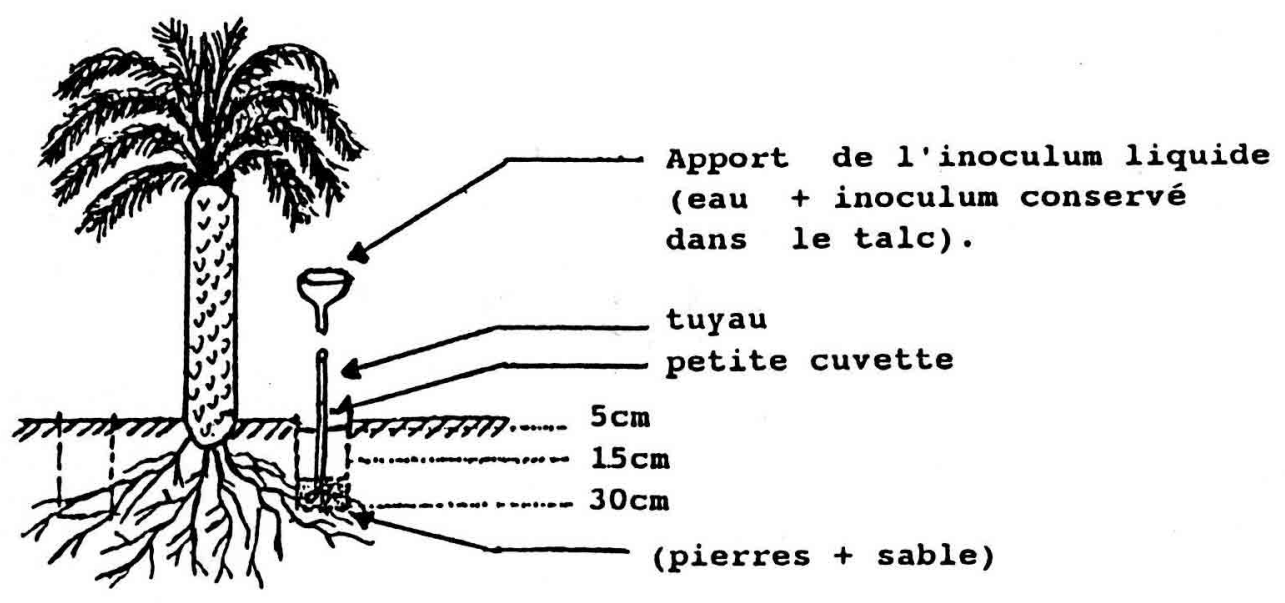

Fig 2. Schéma de la méthode $T$ d'inoculation artificielle des palmiers en plantation (méthode de 2 trous + tuyaux). 
Tableau I. Niveaux du potentiel infectieux et de réceptivité des échantillons de sol du domaine expérimental de Zagora (INRA) à la fusariose vasculaire (bayoud) du palmier dattier exprimés en nombres moyens de plantes mortes par $F$ of $\mathrm{sp}$ albedinis, 12 mois après le semis en pots.

Sols

$\mathrm{ZI}$
$\mathrm{Z} 2$
$\mathrm{Z3}$
$\mathrm{Z4}$
$\mathrm{Z5}$
$\mathrm{Z6}$
$\mathrm{Z7}$
$\mathrm{Z} 8$
$\mathrm{Z9}$
$\mathrm{Z10}$

Effet doses

$0,3^{d}$

0
0
0
0
0
0
0
1
2
0

0
0
0
0
2
0
0
2
3
0

4
2
0
3
5
5
4
9
8
3

\begin{tabular}{rrrrr}
100 & & 1000 & 5000 & 10000 \\
\hline & $\ldots$ & $\ldots$ & &
\end{tabular}

\section{Doses d'inoculum (propagules par $g$ de sol)}

$\begin{array}{rr}8 & 13 \\ 3 & \\ 3 & \\ 5 & \\ 6 & \\ 7 & \\ 7 & 10 \\ 10 & 1 \\ 12 & 14 \\ 4 & \end{array}$

$\begin{array}{rl}13 & 5 \mathrm{bc} \\ 7 & 2,4^{\mathrm{e}} \\ 5 & 1,6^{\mathrm{e}} \\ 5 & 2,6^{\mathrm{de}} \\ 7 & 4^{\mathrm{c}} \\ 7 & 3,8^{\mathrm{cd}} \\ 10 & 4,2^{\mathrm{c}} \\ 11 & 6,6^{\mathrm{b}} \\ 14 & 7,8^{\mathrm{a}} \\ 4 & 2,2^{\mathrm{e}}\end{array}$

$\begin{array}{llll}0,7^{\mathrm{d}} & 4,3^{\mathrm{c}} & 6,5^{\mathrm{b}} & 8,3^{\mathrm{a}}\end{array}$

Les moyennes calculées sur 6 répétitions de 5 plantes chacune et suivies d'une même lettre ne sont pas significativement différentes pour $P=0,05$ (test de Duncan). Effets significatifs des «sols» $(F=17,53)$, "doses" $(F=115,9)$ et leur interaction $(F=2,26)($ analyse de la variance).

\section{RÉSULTATS}

\section{Potentiel infectieux et réceptivité des sols au bayoud}

Le potentiel infectieux des sols est nul dans 8 sols sur 10 et relativement faible ( 1 à 2 plantes

Tableau II. Effet de l'inoculation artificielle sur des palmiers avec Fusarium oxysporum $\mathrm{f} \mathrm{sp}$ albedinis et incidence de la maladie (méthode Ft).

$\begin{array}{lcc}\begin{array}{l}\text { Palmier a } \\ c v \text { Jihel }\end{array} & \text { Nombre } & \begin{array}{c}\text { Nombre de } \\ \text { palmiers atteints }\end{array} \\ \begin{array}{l}\text { Palmiers inoculés } \\ \text { artificiellement }\end{array} & 73 & 12^{\mathrm{b}}(16,4 \%) \\ \begin{array}{l}\text { Palmiers non inoculés } \\ \text { (sol infesté naturellement) }\end{array} & 55 & 2(3,6 \%)\end{array}$

Entre parenthèses : pourcentage cumulé d'attaque. a Palmiers du cv Jihel (sensible) plantés dans les parcelles expérimentales du domaine expérimental de Zagora et inoculés en juillet 1982 avec le broyat de palmes atteintes de bayoud. L'incidence de la maladie exprimée en nombre de palmiers atteints est évaluée après 4 ans d'observation des palmiers au champ (juillet 1986).

b Effet significatif de l'inoculation (test de $\chi^{2}$ ). atteintes sur 30 testées) dans les 2 autres (tableau I). II montre que l'infectivité augmente mais de manière différente en fonction des sols, après infestation artificielle avec les doses croissantes d'inoculum. L'analyse des résultats montre des effets significatifs des «sols», «doses» et leur interaction. En outre, les niveaux de réceptivité des sols sont significativement différents. À la dose 10000 propagules par $\mathrm{g}$ de sol, le nombre moyen de plantes mortes sur 30 testées varie de 4 , pour le sol Z10, à 14 pour le sol Z9. La régression des sols sur les doses d'inoculum n'est pas linéaire ; il s'agit d'une équation parabolique d'ordre 2. Ceci indique que, pour le même sol, le nombre de plantes attaquées à certaines doses, particulièrement 5000 et 10000 propagules par $\mathrm{g}$ de sol, est significativement comparable.

\section{Intérêt de l'inoculation artificielle dans la sélection des palmiers résistants au bayoud}

L'effet significatif de l'inoculation artificielle avec le parasite apporté sous forme de broyat de palmes contaminées $(\mathrm{Fb})$ montre que, sur les 73 plants inoculés artificiellement, 12 sont atteints par la maladie, soit un taux d'attaque de $16,4 \%$. En revanche, sur les 55 plants non inoculés artifi- 
Tableau III. Évolution de l'incidence du Fusarium oxysporum $f \mathrm{sp}$ albedinis sur des palmiers inoculés au champ avec les méthodes Ft et T au domaine expérimental de Zagora (INRA).

\begin{tabular}{|c|c|c|c|c|c|}
\hline \multirow{2}{*}{$\begin{array}{c}\text { Méthode d'inoculation } \\
\text { Traitement }\end{array}$} & \multirow{2}{*}{$\begin{array}{l}\text { Nombre de } \\
\text { palmiers }\end{array}$} & \multicolumn{4}{|c|}{ Nombre de palmiers atteints } \\
\hline & & 1 an & 2 ans & 3 ans & 4 ans \\
\hline \multicolumn{6}{|l|}{ Méthode Ft } \\
\hline cv Jihel inoculés & 29 & 2 & 7 & 15 & $18^{a}(65,5 \%)$ \\
\hline cv Jihel non inoculés & 37 & 4 & 6 & 8 & $8(18 \%)$ \\
\hline cv Bousthami noire inoculés & 6 & 0 & 0 & 0 & $0 \quad(0 \%)$ \\
\hline \multicolumn{6}{|l|}{ Méthode T } \\
\hline cv Jihel inoculés & 10 & 4 & 4 & 7 & $9^{a}(90 \%)$ \\
\hline cv Jihel non inoculés & 10 & 1 & 2 & 3 & $3(30 \%)$ \\
\hline
\end{tabular}

Incidence exprimée en nombre de palmiers atteints après 4 ans d'observations. Entre parenthèses : pourcentage cumulé d'attaque. Méthode F: inoculum apporté après un dégagement des racines (fossé de $50 \mathrm{~cm}$ de profondeur). Méthode $\mathrm{T}$ : inoculum apporté sur les racines au moyen des tuyaux. Tous les palmiers utilisés (cultivars Jihel sensible et Bousthami noire résistant) avaient été déjà inoculés en 1982 avec le broyat de palmes atteintes de bayoứ après 4 ans d'observation. a Effets significatifs des méthodes d'inoculation Ft et T sur l'incidence de la maladie sur les palmiers (test de $\chi^{2}$ ).

Tableau IV. Effet de l'incidence du Fusarium oxysporum $f \mathrm{sp}$ albedinis sur les rejets de palmier plantés, enracinés dans les sachets en plastiques et installés en sachets sous abri (pépinière) et inoculés au moyen des tuyaux en 1989 au domaine expérimental de Zagora (INRA).

Cultivar Nombre de palmiers

Nombre de palmiers atteints en fonction de temps (mois)

Jihel 10

Iklane

10

10
6

6

3

0
9

4

0
12

$4(40 \%)$

$0(0 \%)$

Entre parenthèses : pourcentage cumulé d'attaque. Cultivars sensible (Jihel) et très tolérant (lklane) au bayoud. Les palmiers témoins aveć l'eau ne présentent aucun symptômes de flétrissement.

ciellement, mais placés en sol bayoudé, seuls 2 sont atteints par la maladie soit un taux d'attaque de $3,6 \%$ (tableau II).

\section{Appréciation de l'efficacité des méthodes d'inoculation Ft et $T$}

Après 4 années d'observation au champ, les résultats indiquent (tableau III) les points suivants :

La méthode d'inoculation artificielle dite du "fossé/talc» (Ft), appliquée au cv Jihel, a permis l'expression de la maladie à 18 plants sur les 29 traités $(65,5 \%)$. En revanche, sur le même cultivar non inoculé artificiellement mais localisé sur sol naturellement infesté, seuls 8 plants sur 37 sont attaqués par le bayoud (18\%). Par ailleurs, les 6 plants du cultivar résistant Bousthami noire inoculés artificiellement selon la même méthode (Ft) n'ont manifesté aucun signe de maladie.

La méthode d'inoculation artificielle dite du "tuyau» (T), appliquée au cultivar sensible Jihel, a permis d'infester 9 plants sur $10(90 \%)$ contre 3 plants atteints sur les 10 non artificiellement inoculés, mais localisés sur sol naturellement infesté $(30 \%)$.

\section{Intérêt de la mise au point d'une méthode d'inoculation des rejets installés en sachets}

La méthode d'inoculation des rejets installés en sachets a permis, après seulement une année, 
de provoquer la mort de 4 jeunes palmiers sur les $10 \mathrm{du} \mathrm{cV}$ Jihel développés sous abri (tableau IV). Cette même méthode appliquée au cv Iklane très tolérant n'a entraîné aucun signe de dépérissement. Les palmiers témoins des 2 cultivars n'ont manifesté aucun signe de maladie.

\section{DISCUSSION}

Il est admis que la densité d'inoculum comme sa répartition dans un sol infesté naturellement jouent un rôle déterminant dans la rapidité de développement des maladies telluriques. Dans le cas du bayoud, les premières inoculations expérimentales que nous avons réalisées (Djerbi et Sedra, 1982) avec le broyat de palmes atteintes ont montré que l'on peut augmenter le potentiel infectieux des sols, des sites expérimentaux, en élevant la densité du parasite et en homégénéisant sa répartition. Ces observations sont confirmées au laboratoire par des tests d'appréciation du potentiel infectieux et de réceptivité des sols. Ceci explique l'hétérogénéité d'infection constatée au domaine expérimental de Zagora et rend difficile l'interprétation des résultats obtenus en matière de sélection des palmiers résistants soumis à la pression de l'inoculum naturel. En effet, les échantillons de sols Z2, Z3 et Z10 présentent globalement un niveau très faible de receptivité à des doses d'inoculum faibles à peu élevées. Ceci peut probablement expliquer les différences de comportement à l'égard du bayoud des palmiers témoins sensibles observées par Saaidi (1992), dans certains blocs du domaine de Zagora. L'analyse globale de nos résultats montre que les effets «sol» et «dose» sont significatifs ainsi que leur interaction. La régression de la réponse de ces échantillons de sol à l'accroissement des doses n'est pas linéaire mais quadratique. Or, le fait que le potentiel infectieux d'un sol soit égal à 0 n'est pas toujours dû aux phénomènes de réceptivité de ce sol mais elle peut être simplement due à l'absence de l'inoculum naturel ou à une insuffisance de sa densité (tableau I). Les résultats de la réceptivité confortent cette constatation. Ces 2 paramètres - connaissance du potentiel infectieux et réceptivité des sols - sont nécessaires dans un programme de sélection et d'étude de comportement des cultivars à l'égard du bayoud. La comparaison des lots de palmiers inoculés, artificiellement ou non, montre bien l'intérêt des inoculations expérimentales. Ces inoculations ont fait la preuve de leur efficacité dans les parcelles expérimentales d'Errachidia qui n'ont jamais porté de palmier (Sedra, 1993b).
Cependant, plusieurs séries d'analyse micro-biologique dans les sols des domaines expérimentaux de Zagora et d'Errachidia (résultats non publiés), inoculés avec le broyat de palmes atteintes, ont permis de conclure que la densité du parasite diminue rapidement en fonction du temps. Cette diminution peut être due à la faible aptitude du parasite à la colonisation saprophytique face aux actinomycètes, bactéries et autres microorganismes qui exploitent facilement le broyat de palmes. L'utilisation du talc comme support physico-chimiquement inerte et facilement diluable a permis de mieux contrôler les apports d'inoculum (Alabouvette et al, 1982).

Bien que l'efficacité des méthodes $\mathrm{Ft}$ et $\mathrm{T}$ ait été démontrée (tableau II), elles présentent certains avantages et inconvénients : la méthode $\mathrm{Ft}$ permet à l'inoculum d'être mis efficacement en contact d'un grand nombre de racines d'un palmier mais elle exige pour l'appliquer des travaux considérables traumatisants.

La méthode $\mathrm{T}$ semble plus efficace et plus simple d'application, nécessitant moins de travaux destructeurs de racines que ceux fournis pour les méthodes $\mathrm{Fb}$ et $\mathrm{Ft}$. Elle permet de s'adapter aux conditions d'inoculation des jeunes palmiers cultivés en hors-sol. II est important de noter que toutes ces méthodes nécessitent un contrôle régulier de la densité du parasite qui peut diminuer lentement ou rapidement selon les capacités d'accueil des sols où s'effectue une telle sélection.

La sélection en palmeraies faite depuis plus de 20 ans sur le critère de résistance est relativement lente. En effet, pour le cv Jihel reconnu sensible au bayoud par les agriculteurs, Saaidi et a/ (1991) ont obtenu un pourcentage moyen d'attaque de $36 \%$ après 13 années et de $50,6 \%$ (Saaidi, 1992) après 25 années d'observation sur le terrain infesté naturellement au domaine expérimental de Zagora. Après seulement 4 années d'observation dans le même site, nous avons relevé, pour ce même cultivar, $65,5 \%$ et $90 \%$ d'attaque respectivement avec les méthodes $\mathrm{Ft}$ et $T$ d'inoculation artificielle. Ceci montre qu'il est possible de réduire de cinq à six fois le temps de sélection au champ.

L'inoculation des jeunes palmiers cultivés en hors-sol (sachet contenant du sable et de la terre de Zagora (2v/1v)) est encore plus efficace puisqu'en une année 4 rejets sur $10 \mathrm{du} c v$ Jihel ont été attaqués. Les palmiers témoins des 2 cultivars (Jihel et Iklane) n'ont manifesté aucun signe de flétrissement. L'inoculation des jeunes palmiers sous abri est simple, peu encombrante, 
facilement maîtrisable et peu coûteuse. Elle permet d'évaluer la résistance de plusieurs séries de clones et cultivars en même temps nécessitant seulement quelques rejets de chaque génotype, pour un tri préliminaire avant confirmation de la résistance sur vitroplants (Sedra, 1993b). De plus, la nécessité de cette présélection permet de proposer un nombre raisonnable de clones pour la multiplication in vitro selon les capacités des laboratoires de culture des tissus.

\section{REMERCIEMENTS}

Je remercie le professeur M Besri (IAV Hassan-II, Rabat, Maroc), les docteurs F Rouxel (INRA, Le Rheu, France) et $R$ Loussert (INRA, Marrakech, Maroc) pour leurs remarques précieuses et suggestions enrichissantes. Mes remerciements vont également aux techniciens du laboratoire et particulièrement $D$ Frira et tout le personnel du domaine expérimental de Zagora (INRA) qui m'ont aidé à réaliser ce travail. Je tiens à remercier également le projet PNUD/FAO de lutte contre le bayoud et la Fondation internationale pour la science (IFS) pour leur soutien financier de mon projet de recherche.

\section{RÉFÉRENCES}

Alabouvette C, Couteaudier Y, Louvet J (1982) Comparaison de la réceptivité de différents sols et substrats de culture aux fusarioses vasculaires. agronomie, 2, 1-6

Bulit J, Louvet J, Bouhot D, Toutain G (1967) Recherches sur les fusarioses. I. Travaux sur le bayoud, fusariose vasculaire du palmier dattier en Afrique du Nord. Ann Epiphyties 18, 231-239

Djerbi M (1988) Les maladies du palmier dattier. Projet régional de lutte contre le bayoud. FAO (Rab/84/018), Alger, $127 \mathrm{p}$.

Djerbi M, Sedra My H (1982) Screening commercial Iraqi date varieties to bayoud. Nenadates News $n^{\circ} 2,1$, FAO

Djerbi M, Sedra My H, El Idrissi MA (1985) Caractéristiques culturales et identification du Fusarium oxysporum $\mathrm{f} s \mathrm{sp}$ albedinis, agent causal du Bayoud. Ann Inst Nat Rech Agr Note de Rech, $\mathrm{n}^{\circ}$ 1, 58, 1-8

Djerbi $M$, Aouad $H$, El Filali $H$, Saaidi $M$, Chtioui $A$, Sedra My H, Allaoui M, Hamdaoui T, Oubrich M (1986) Preliminary results on selection of high quality bayoud resistant clones among natural date palm population in Morocco. In: The second symposium on date palm, 3-6 mars 1986, univ Roi-Faïssal, Arabie Saoudite, 386-399

Louvet J, Toutain G (1973) Recherches sur les fusarioses. VII. Nouvelles observations sur la fusariose du palmier dattier et précisions concernant la lutte. Ann Phytopathol 5, 35-52

Louvet J, Bulit J, Toutain G, Rieuf P (1970) Le bayoud, fusariose vasculaire du palmier dattier : symptômes et nature de la maladie : moyens de lutte. Al Awania, 35, 161-181

Pereau-Leroy $P$ (1958) Le palmier dattier au Maroc. Ministère de l'Agriculture, Instit Franc Rech Outremer, Paris, $142 \mathrm{p}$

Saaidi M (1992) Comportement au champ de 32 cultivars de palmier dattier vis-à-vis du bayoud : 25 ans d'observations. agronomie, 12, 259-270

Saaidi M, Toutain G, Bannerot H, Louvet J (1981) La sélection du palmier dattier (Phoenix dactylifera $\mathrm{L}$ ) pour la résistance au bayoud. Fruits 4, 36, 241249

Sedra My H (1985) Potentiel infectieux et réceptivité de quelques sols de palmeraie à la fusariose vasculaire de palmier dattier (bayoud) causée par Fusarium oxysporum $\mathrm{f} \mathrm{sp}$ albedinis (Malençon) $\mathrm{Sn}$ et $\mathrm{H}$. Thèse $3^{e}$ cycle, Inst agro vétér Hassan-II, Rabat, Maroc, $88 \mathrm{p}$

Sedra My H (1990) Preliminary results on the evaluation of the resistance to the bayoud of the clone (Khalts), cultivars and some hybrids of the date palm trees selected on the fruit quality criterium. In : 8th Cong of Mediterr Phytopathol Union, 28 oct - 3 nov 1990, Agadir, Maroc

Sedra My H (1992) Evaluation and selection of the resistant good cultivars and clones of the date palm to the bayoud disease. Arab Soc Plant Protect, 10, 2, 155-160 (texte en arabe, abstract en anglais)

Sedra My H (1993a) Remarques sur le pouvoir pathogène des isolats de Fusarium oxysporum $\mathrm{f} \mathrm{sp}$ albedinis, agent de la fusariose vasculaire (bayoud) du palmier dattier. Al Awamia 83, 210-223

Sedra My H (1993b) Lutte contre le bayoud, fusariose vasculaire du palmier dattier causée par Fusarium oxysporum $\mathrm{f} \mathrm{sp}$ albedinis : selection des cultivars et clones de qualité résistants et réceptivité des sols de palmeraies résistants à la maladie. Thèse doct État ès-sciences, univ Cadi-Ayyad, Marrakech, Maroc, $142 \mathrm{p}$

Sedra My H, Rouxel F (1989) Résistance des sols aux maladies. Mise en évidence de la résistance d'un sol de la palmeraie de Marrakech aux fusarioses vasculaires. Al Awamia 66, 35-54

Sedra My H, Besri M, Rouxel F (1994) Caractérisation des niveaux de réceptivité des sols de palmeraie marocaine aux fusarioses vasculaires, en particulier le bayoud. Phytopathol Mediter 33, 27-35

Tello-Marquina JC, Alabouvette C (1984) Observation sur la persistance dans le sol des microconidies de Fusarium oxysporum. agronomie 4, 885-890

Toutain G (1977) Élements d'agronomie saharienne. De la recherche au développement. Jouve, Paris, $277 \mathrm{p}$ 